\title{
Supercritical Fluid Extraction of Lycopene and Omega-3
}

\author{
MARIA MARGOTTA \\ Meid4 Academic Spin-Off of the University of Salerno \\ Via Giovanni Paolo II, 132, 84084 Fisciano SA, ITALY \\ maria_margotta@meid4.it \\ DOMENICO GUIDA \\ Department of Industrial Engineering - University of Salerno \\ Via Giovanni Paolo II, 132, 84084 Fisciano SA, ITALY \\ guida@unisa.it
}

\begin{abstract}
An ideal extraction method should be rapid, produce a quantitative recovery without degradation and the extracts should be easily separated from the solvent. Supercritical fluid technology offers features that overcome many limitations of conventional extraction methods. There are many advantages to their application, especially in the food sector (for example the extraction of lycopene from tomato skins or the extraction of fish oil rich in omega-3 from fish by-products); the extraction method influences the yield and quality of the product obtained, and also allows to operate with moderate temperatures and with shorter timescales. The processes involving SCF are sustainable, environmentally friendly and cost efficient, and offer the possibility of obtaining new products. The disadvantage, however, lies in the fact that it is necessary to use expensive equipment and to perfect the particular phases of the process to obtain a specific product.
\end{abstract}

Key-Words: - Supercritical Fluids Extraction, Supercritical $\mathrm{CO}_{2}$, Supercritical Fluids Fractionation, Lycopene, Omega-3, Polyunsaturated Fatty Acids.

Received: August 17, 2019. Revised: December 27, 2019. Accepted: January 29, 2020. Published: January 7, 2020.

\section{Introduction}

The application of supercritical fluids as an alternative to conventional processes of precipitation, separation and extraction it is a field of research in continuous innovation.

The use of supercritical $\mathrm{CO}_{2}$ offers innumerable advantages, including moderate operating temperature, density and solvent power that can be easily adjusted thanks to a minimum change in temperature or pressure, flammability and low cost. Nowadays, $\mathrm{SC}-\mathrm{CO}_{2}$ is used for large-scale extractions in the food industry, as an alternative to traditional separation systems, such as fractional distillation, steam current extraction, extraction with organic solvents.

A large application of supercritical fluids occurs in the agro-industrial field, above all for the recovery of nutrients from processing waste. In fact, in the last few years the concept of "zero waste" has become increasingly aware: waste can be reused within the food production chain. Many residues, in fact, are rich in bioactive species.[1]

The aim of this work is to illustrate how supercritical $\mathrm{CO}_{2}$ is able to extract lycopene from tomato production waste and omega-3 rich oil from fish by-products.

\section{Supercritical Fluids Properties}

When the substance is above its critical temperature and pressure, the fluid is called supercritical. This fluid has properties similar to gas and liquid: it is similar to gas because it is a compressible fluid and is similar to the liquid for the comparable density value. [2]

It is possible to show the dependence of solubility

$(\delta)$ with density $(\rho)$ using the Hildelband formula:

$$
\delta=1.25 P_{c}^{0.5}\left(\begin{array}{c}
\rho \\
\rho_{l i q}
\end{array}\right)
$$

With this formula the density is seen as a property that measures the solvent power of the supercritical fluid. With small variations of pressure, the fluid changes its density and, consequently, its solvent power.

Other important properties are the viscosity and the diffusion coefficient: the speed with which a fluid is able to interact with a specific solute depends on them. The transport properties for a supercritical 
fluid are particularly favorable, since they are closer to the properties of the gaseous state rather than to those of the liquid state.

Extraction with a supercritical fluid of comparable solvent power is therefore faster, a further reason why the use of supercritical fluids in different industrial sectors continues to be tested more and more.

The advantages of extraction with supercritical fluids (compared to extraction with liquids) are the speed of the operation, given the low viscosity and high diffusivity of SCFs, the high commercial value of the extracted product and purity, and the disengagement from environmental limitations.

The solvent capacity of a supercritical fluid is comparable to a liquid of equal polarity but, unlike liquids, it is able to penetrate, like a gas, inside the solid matrix. As regards the extractive selectivity of the supercritical fluid, the fractionation of liquids and solids can be obtained if the constituents of the matrix to be fractionated differ in volatility (the compounds with higher vapor tension are more soluble), mass (the pressure necessary for the 'extraction increases with the molecular weight of the compounds) and polarity (for example, in a nonpolar supercritical fluid such as $\mathrm{CO}_{2}$ the solubilization of apolar compounds is favored).

\subsection{Characteristics of Supercritical $\mathrm{CO}_{2}$}

The most used supercritical fluid is carbon dioxide: it is inexpensive, chemically inert, non-toxic, nonflammable and readily available at high purity and low cost. Furthermore, the critical point of the $\mathrm{CO}_{2}$ is easily reachable (critical temperature $31.1^{\circ} \mathrm{C}$ and critical pressure 73.8 bar) which allows using the fluid at average temperatures $\left(40-60{ }^{\circ} \mathrm{C}\right)$ without leaving harmful organic residues. [3]

The choice of the type of fluid to be used in a specific process depends on technological, economic and environmental impact considerations: it must have relatively low critical temperature and pressure values, it must also be easily separable from other materials present at the end of the process, it must not be flammable or toxic, and have a low cost.

In Table 1 there are some fluids that can be used in supercritical conditions.

\begin{tabular}{|l|l|l|}
\hline Fluid & $\mathbf{T c}\left[{ }^{\circ} \mathbf{C}\right]$ & Pc $[$ bar $]$ \\
\hline Carbon Dioxide & 31.1 & 73.8 \\
\hline
\end{tabular}

\begin{tabular}{|l|l|l|}
\hline Difluoromethane & 111.8 & 39.0 \\
\hline Trifluoromethane & 28.8 & 39.0 \\
\hline Ethane & 32.2 & 48.8 \\
\hline Ethylene & 9.3 & 50.4 \\
\hline Propane & 96.7 & 42.5 \\
\hline Propylene & 91.8 & 45 \\
\hline $\mathbf{H}_{2} \mathbf{O}$ & 374.2 & 220.5 \\
\hline $\mathbf{N}_{\mathbf{2}} \mathbf{O}$ & 36.5 & 71.7 \\
\hline
\end{tabular}

Table 1: Possible supercritical solvents and related critical temperature and pressure parameters.

\section{Processes Assisted by Supercritical Fluids}

The application of supercritical fluids as an alternative to conventional processes of precipitation, separation and extraction is a field of research in continuous innovation. The main reason is the possibility of exploiting the peculiar characteristics of supercritical carbon dioxide. [1] The processes that use supercritical $\mathrm{CO}_{2}$ include:

SFE (SupercriticalFluidsExtraction): it can replace many traditional extraction processes from vegetable matrices to obtain dry extracts or essential oils with specific characteristics;

$>$ SFF (SupercriticalFluidsFractionation): unlike the SFE process, the matrix which to extract is in the liquid phase;

\section{$>$ RESS}

(RapidExpansionOfSupercriticalSolution): generally used to produce fine particles for the food, cosmetic and pharmaceutical industries;

$>$ PGSS

(ParticlesFromGasSaturatedSolution):

microparticles are produced, but unlike the RESS process, the matrix is first liquefied and then solubilized with $\mathrm{SC}-\mathrm{CO}_{2}$ : a solution or a suspension saturated with gas is created which is then depressurized up to the environmental conditions;

> SAA (SupercriticalAssistedAtomizatio): the liquid solution is saturated with $\mathrm{CO}_{2}$ in a packed column before its dispersion. 
$>$ SAS (SupercriticalAntiSolvent): The principle is to put in contact an organic solution containing the solute to be precipitated with a supercritical fluid, such as the supercritical $\mathrm{CO}_{2}$, which acts as an anti-solvent for the solute but is completely miscible with the organic solvent.[4]

$>$ SEE (SupercriticalExtractionOfEmulsion): Using this technique, unlike conventional evaporation of the emulsion solvent, are obtained particles which extract the organic solvent of the oily phase very quickly using supercritical carbon dioxide $\left(\mathrm{SC}-\mathrm{CO}_{2}\right)$

\subsection{SFE (SupercriticalFluidsExtraction from Natural Solid Matrices)}

SFE is mainly used in the food field, for example in the decaffeination of coffee, in the extraction of flavors and aromas (essential oils, vanilla, chamomile, spices) and also in the extraction of fats, oils, cholesterol from food products; it is used in the pharmaceutical field because as a result of these extractions there are no traces of toxic solvents and therefore the medicines have fewer side effects.

The SFE is applied in the environmental field, in the extraction of contaminants from industrial waters and wastewaters, in the petrochemical sector for the fractionation of heavy oils but also in the commercial sector.

One of the fields where most experiments have been done with the use of supercritical fluids is the extraction from vegetable matrices.

The extraction of substances from complex mixtures, in particular, can be made highly selective modifying the pressure and temperature conditions, to adapt them to the solubility of the different components of specific interest.

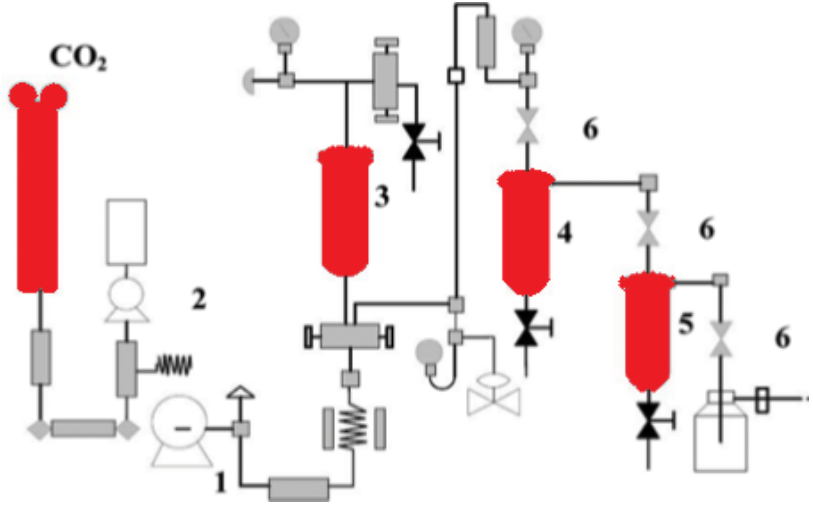

Fig. 1: Design of a pilot extraction plant with supercritical fluid equipped with two fractionation cells
(1) $\mathrm{CO}_{2}$ pump; (2) co-solvent or modifier pump; (3) extraction cells for solid samples; (4) fractionating cell; (5) fractionating cell 2 ; (6) valves

The extraction cells and the separators of the system are generally provided with independent temperature and pressure regulators so that the fractionation of the compounds extracted from the matrix can be performed by modifying the operating systems. Separators placed in series can be applied in different conditions and therefore different compounds can be separated depending on the solubility of the compound in the supercritical fluid (given to the condition applied in that separator).

The main difference between the pilot plants for the treatment of solid or liquid samples is the use of an extraction cell or an extraction column respectively. The processing of the solid is always carried out in batch and in a non-continuous or semi-continuous process and, generally, the processing of the liquids is carried out continuously in countercurrent conditions.

The selection of the operating conditions of the SFE process is mainly based on the solubility (of the solute) in the solvent and/or on the overall extraction efficiency, which is defined as the maximum quantity of material that can be extracted at a certain temperature, pressure and outflow.

\subsubsection{Supercritical $\mathrm{CO}_{2}$ Extraction of Lycopene from Tomatoes in the Presence of Vegetable Oil as a Co-Solvent}

Lycopene $\left(\mathrm{C}_{40} \mathrm{H}_{56}\right)$ is the carotenoid pigment most present in tomatoes (it represents about $85 \%$ of the total carotenoids) and gives it its characteristic red color. Its presence varies considerably depending on the variety, degree of ripeness, environmental conditions and cultivation techniques. In mature tomatoes typical lycopene values of $50 \mathrm{mg} / \mathrm{kg}$ are found, but in some varieties this level can even exceed $70 \mathrm{mg} / \mathrm{kg}$.

The main problem in obtaining lycopene is its solubility and stability: it is insoluble in water but soluble in highly toxic organic solvents, such as benzene, chloroform, dichloromethane and decomposes easily over time. In order to overcome these difficulties and obtain lycopene without traces of organic solvents, the scientists considered the use of the extraction technique with supercritical fluids (SFE).

Experiments conducted with and without co-solvent at pressures and temperatures between 335 and 450 bar and $45-70{ }^{\circ} \mathrm{C}$, respectively, have shown that the quantity of lycopene that can be extracted depends 
on the experimental conditions. Furthermore, the maximum quantity of lycopene extractable from the dried tomato $(6 \%$ humidity, average grain size about $1 \mathrm{~mm}$ ), at 450 bar and $66^{\circ} \mathrm{C}$ in the presence of cosolvent and using a flow rate about $20 \mathrm{~kg}$ of $\mathrm{CO}_{2} / \mathrm{h}$, it was $60 \%$. [5]

The presence of the co-solvent improves the yields of the lycopene extract and has a beneficial role in pigment stability.

Various vegetable oils have been tested (almond oil, peanuts, hazelnuts and sunflower seeds) but only in the case of hazelnut oil (cheaper and its low acidity prevents the degradation of lycopene during extraction) have been obtained higher extraction yields. A greater quantity of oil provides a more diluted extract of lycopene and for this reason the average value of $10 \%$ was chosen.

Since there is a high degree of humidity in tomatoes, the raw material was initially dried and then ground to obtain significant yields of lycopene. With smaller particle sizes, the ratio surface/volume of the sample increases and therefore the contact between the solid phase and the liquid phase increases. For this reason, an average particle size of $1 \mathrm{~mm}$ was chosen.

Extraction with $\mathrm{SC}-\mathrm{CO}_{2}$ was performed with the apparatus shown in Fig. 2, equipped with an AO1 extractor (10 1) and three separators S1 (1.5 1), S2 (1.5 1) and $\mathrm{S} 3$ (0.3 1) in series. The $\mathrm{CO}_{2}$, pressurized to the desired level by the P1 pump (Milton Roy), flows through a heating coil (E2) before reaching the AO1 extraction bed.

Then, leaving AO1, the fluid subsequently flows towards the separator S1 through a cooling coil (E3) and then towards the separator S2 passing through a micro-measurement valve (VL1) with consequent reduction of the pressure and, therefore, reduction of the density of $\mathrm{CO}_{2}$.

The solute that precipitates from the stock solution can be collected in S2. The fluid subsequently arrives in the separator S3 through the second micrometric valve (VL2) with a reduction of pressure up to its final value and the solute is then separated and collected in $\mathrm{S} 3$. The $\mathrm{CO}_{2}$ that comes out of the S3 separator can be recycled by passing through the F1 filter and the E1 condenser.

The S1 separator operates with constant pressure; the variation of the temperature reduces the solubility of the extract, producing the separation and its precipitation. In the separators S2 and S3 the solubility decreases with the variation of the pressure.

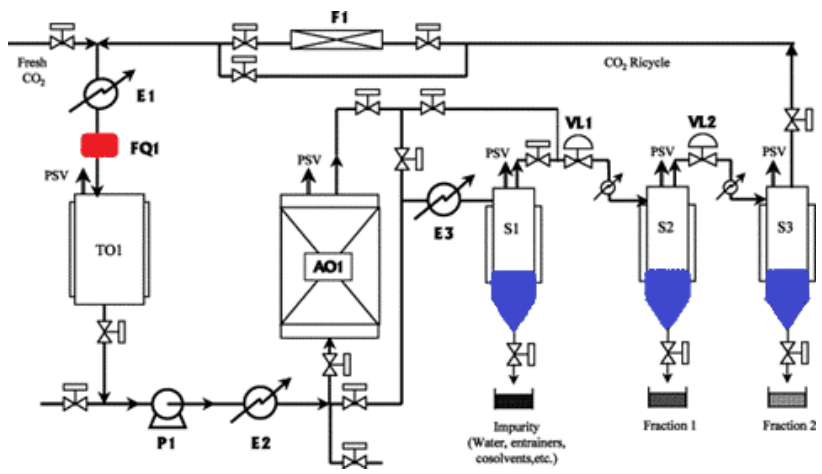

Fig. 2: SFE apparatus

AO1, extractor; S1-3, separators; $\mathrm{TO} 1, \mathrm{CO}_{2}$ supply tank; F1, filter; P1, pump; E1, condenser; E2, heating battery; E3, cooling coil; VL1-2, micrometric valves; FQ1, flow meter; PVS, safety valves.

Several experiments were performed varying the Pressure and Temperature and it was noted that:

$>$ An increase in the Pressure, working with a constant Temperature, increases the density of the solvent and therefore the solubility of the lycopene and at higher densities the molecular interactions between the solvent and the solute are enhanced, resulting in a greater dissolution of the solute.

An increase in temperature, working at constant Pressure, reduces the density of the solvent and, consequently, reduces the solubility of lycopene but promotes the transport of the solute from the matrix to the solvent.

The best operating conditions experimentally achieved are the following:
$>\mathrm{P}=450$ bar
$>\mathrm{T}=65-70^{\circ} \mathrm{C}$
$>\mathrm{CO}_{2}$ mass flow rate $=18-20 \mathrm{~kg} / \mathrm{h}$
$>$ Average grain size of the material $=$ approximately $1 \mathrm{~mm}$
$>$ Quantity of hazelnut oil $=10 \% \mathrm{w} / \mathrm{w}$

The optimization of the parameters in the extraction with SC-CO2 as temperature, pressure, flow rate, extraction time, as well as the moisture content and particle size, leads to higher yields of lycopene.

The presence of the co-solvent probably promotes better transport and a better solubility of the pigment from the solid phase to the supercritical phase and allows it to avoid the degradation of lycopene and keep the pigment stable over time. 


\subsection{SFF (Supercritical Fluids Fractionation of the Liquid Feed)}

The SFF fractionation process foresees, as for the previous one, the extraction of a substance with a supercritical fluid, in this case however the matrix consists of a liquid mixture. It is considered an improvement, in terms of yield, of separation processes such as distillation, absorption, and liquidliquid extraction. [6]

The process is carried out in absorption columns with countercurrent fluids. If the molecule to be extracted is chemically very similar to those present in the mixture, the column must be higher. (Fig. 3)

In this case, the choice of process temperatures depends on the critical temperature of the solvent while in the case of distillation, it depends on the liquid-vapor equilibrium conditions of the feed mixture. With the SFF process it is possible to perform difficult separations and at the same time, operate at moderate temperatures.

The block diagram in Fig. 3 shows an SFF extraction plant with supercritical $\mathrm{CO}_{2}$.

The liquid mixture is fed from the top, while the $\mathrm{CO}_{2}$ is blown from below. In the column, the liquid comes into contact with the supercritical fluid that extracts the components with lower molecular weight, while the compounds with a higher molecular weight are found in the liquid bottom stream. Part of the outgoing current in the head is recycled to increase the yield, while the remaining part is separated from the $\mathrm{CO}_{2}$ by decompression.[6]

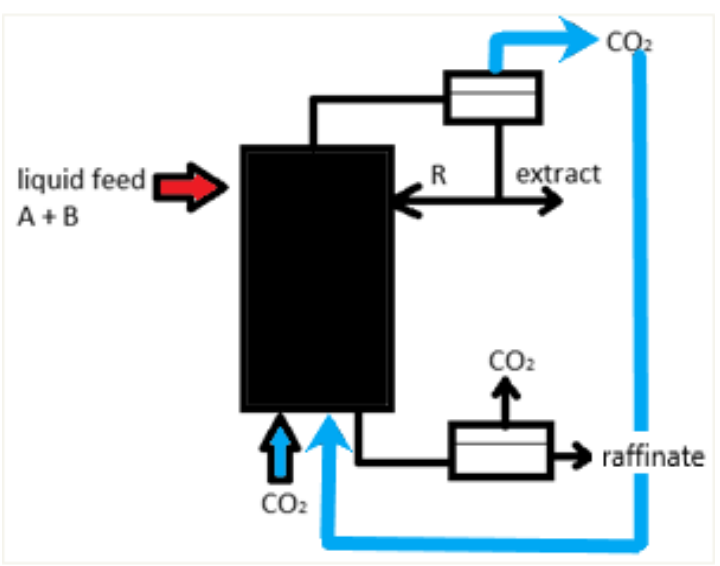

Fig. 3: Process diagram of a counter-current fractionation system with a supercritical fluid for the separation of two components
3.2.1 Extraction of fish oil rich in omega-3 from fish by-products: a comparison, in terms of oil properties, between the different extraction methods

Fish oil is the most generous food source of omega3 , in particular, of eicosapentaenoic acid (EPA) and docosahexaenoic acid (DHA). They are polyunsaturated fatty acids that exert a strong positive influence on human health.

Fish, like men, are not able to directly synthesize omega-3s but accumulate them in their tissues by absorbing them from microalgae or from the animals they eat.

The synthesis of DHA occurs in phytoplankton and animals, but not in plants: DHA is absent from foods of plant origin, including vegetable and oil fats, cereals, nuts, and seeds. However, it is present in the lipids of animal tissues, with the richest source of fatty fish. Meat and milk from ruminants also contain small amounts of DHA.

The production of fish oil takes place mainly through the so-called "wet reduction process": whole fish or fish by-products (mainly viscera, heads and spines) are treated by grinding, heating and centrifuging in one or two stages.

This process makes it possible to obtain high volumes of raw fish oil, although in order to make the oil suitable for edible purposes, subsequent refining phases are necessary.

Four different extraction methods were evaluated to obtain fish oil rich in omega-3:

$>$ cold extraction (CE) or centrifugation

$>$ wet reduction (WR)

$>$ enzymatic extraction (EE)

$>$ supercritical fluid extraction (SFE).

The quantity of raw material used in each extraction method was about $100 \mathrm{~g}$. 


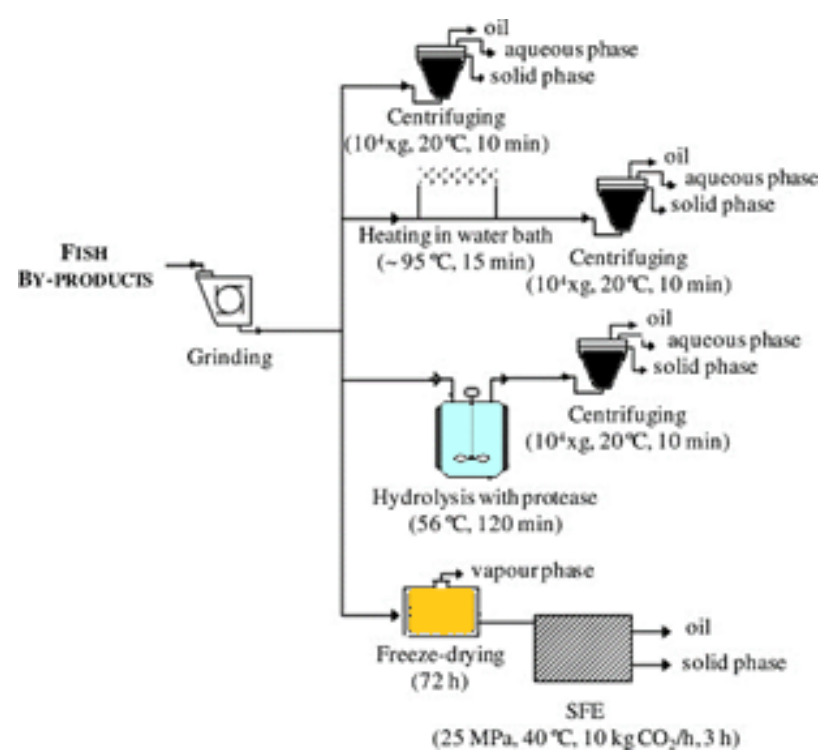

Fig. 4: Scheme of the different fish oil extraction procedures

In the cold extraction, in the wet reduction and in the enzymatic extraction, the fish waste was previously thawed at room temperature for 12 hours and the co-extracted water together with the oil was removed by centrifugation.

For enzymatic extraction the enzyme used was a food-grade protease, Alcalase $2.4 \mathrm{~L}$ (bacterial protease from Bacillus licheniformis).

For the SFE, it was performed in a semi-pilot plant; the raw material used for this extraction method was previously freeze-dried (lyophilized).

The quality of the oil obtained with the different extraction methods was evaluated by determining several parameters:

$>$ moisture content and volatile matter

$>$ neutral lipid composition

$>$ fatty acid profile

$>$ acidity value

$>$ peroxide value

$>$ traces of metals

Extraction with $\mathrm{SC}-\mathrm{CO}_{2}$ allows obtaining fish oil with a high quantity of omega-3 fatty acids, using a non-oxidizing atmosphere and mild temperatures, which prevent the oxidation of polyunsaturated fatty acids.

Several extraction tests were carried out in different fish by-products (off cuts from hake, orange roughly, salmon and livers from jumbo squid) and it was observed that, at the beginning of the process, the extracted oil had a linear dependence with the amount of $\mathrm{SC}-\mathrm{CO}_{2}$ flowing through the extractor, which may indicate that the internal mass transfer is negligible and that the process is controlled by the solubility of the oil in $\mathrm{SC}-\mathrm{CO}_{2}$ or that the internal mass transfer is constant and the extraction rate depends on the internal structure of the solid matrix. The values of the slopes of the extraction curves estimated at time zero are different for each fish product examined, which can be attributed not only to the different internal structure, but also to the different solubility of fish oil in $\mathrm{SC}-\mathrm{CO}_{2}$.

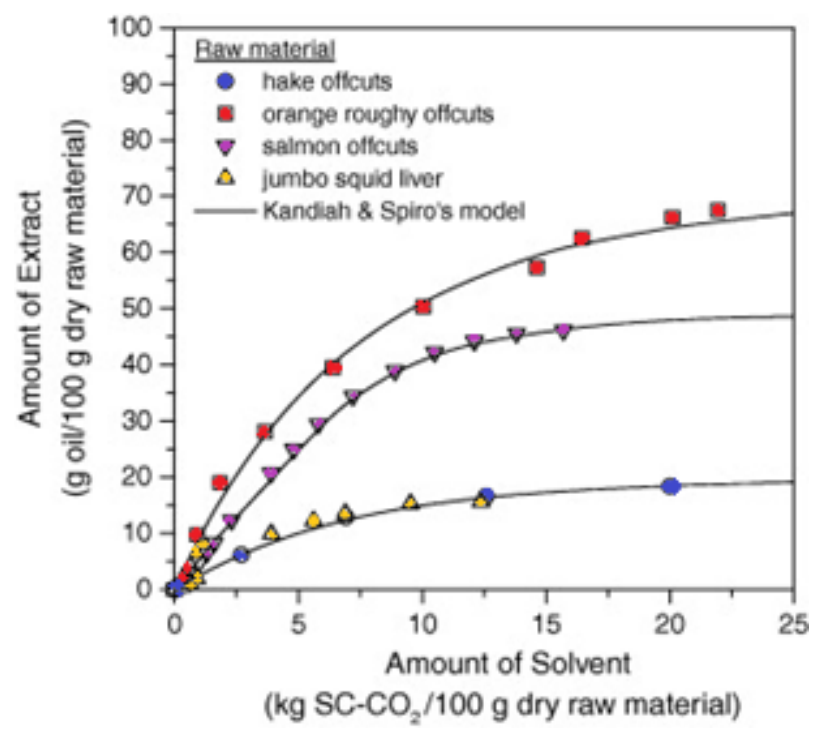

Fig. 5: Extraction curves obtained for SFE of oil from different fish by-products.

The different extraction curves obtained, correctly followed the empirical model of Kandiah and Spiro (1990): the process is controlled by two diffusion stages depending on the quantity of oil accessible to the $\mathrm{SC}-\mathrm{CO}_{2}$. As can be seen, at the beginning the most accessible quantity of oil is high since there is low internal resistance to mass transfer.

As can be seen in Fig. 5, cold extraction was the simplest way to obtain fish oil because it involves only a mechanical phase separation (solid, water and oil) by centrifugation.

The following figure shows an estimate of the mass balance that is obtained when oil is extracted from salmon waste with the four different extraction methods. 


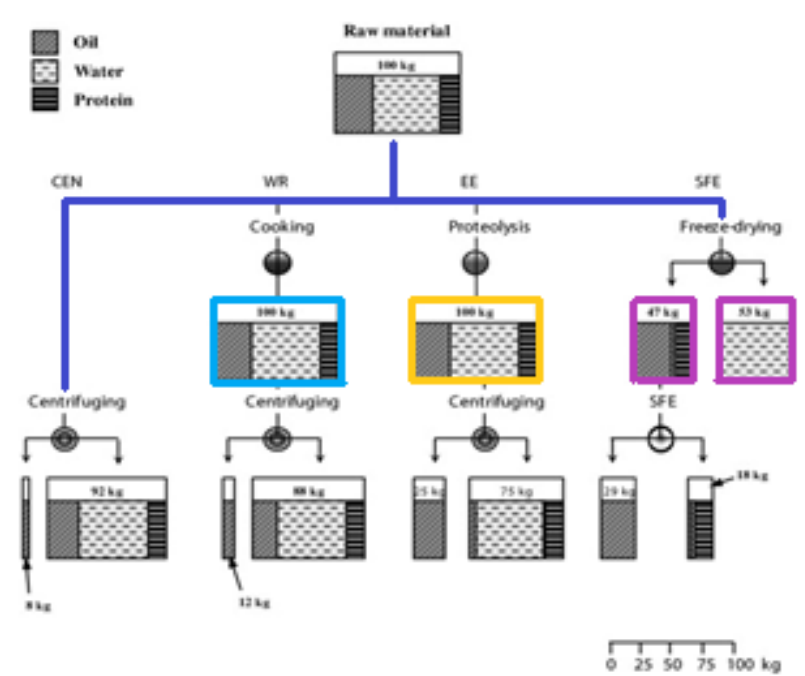

Fig. 6: Estimation of the mass balance that results when obtaining oil from salmon offcuts by the four different extraction methods

In Fig. 6 can be observed that SFE coupled with freeze-drying generates oil with a dry solid phase, rich in protein (fishmeal), while when cold extraction or wet reduction is used, a high amount of oil still remains in the wet solid obtained after centrifugation. This solid would require further treatment in order to obtain a dry, degreased fish meal.

When the enzymatic method is used, almost the total amount of oil can be separated from the aqueous phase containing the protein hydrolyzed by the action of the protease.

A comparison was made between the advantages and disadvantages of the different extraction methods used, comparing the acidity of the oil, the total oxidation value (TOTOX), the volatile compounds, the sensory properties and the heavy metal content because the color, the neutral lipid composition and the fatty acid profile were similar, regardless of the extraction method used.

As for the acidity content, the oil obtained with the SFE technique has a lower acidity than the oil obtained with different techniques compared to SFE.

The TOTOX value is therefore intrinsically related to the amount of PUFA in the oil and to the extraction procedure: the SFE method, allowed to significantly reduce the value of TOTOX.

The sensory properties related to the smell and taste of fish oil strongly depend on the presence of volatile compounds such as organic acids, amines or aldehydes, which are the main responsible for the main fish aromas.

These volatile compounds, present in the raw material, can be easily extracted with $\mathrm{SC}-\mathrm{CO}_{2}$ due to their high vapor pressure and, since the process takes place in a solvent recirculation system. In any case, the amount of these volatile compounds can be significantly reduced by coupling SFE with other separation processes such as fractionation or countercurrent adsorption.

Due to water pollution many toxic compounds accumulate in some parts of the fish: so-called heavy metals, such as $\mathrm{As}, \mathrm{Cd}, \mathrm{Hg}$ and $\mathrm{Pb}$.

Thanks to the high selectivity of SC- $\mathrm{CO}_{2}$ for nonpolar compounds, the amount of heavy metals extracted together with the SFE oil was negligible.

The SFE is followed by the fractionation of the extract into two separators by depressurization: the first separator (S1), maintained at a pressure of $9 \pm$ $0.5 \mathrm{MPa}$ and a temperature of $308 \pm 1 \mathrm{~K}$, and the second separator (S2), maintained at a pressure of 5 $\pm 0.5 \mathrm{MPa}$ and a temperature of $283 \pm 1 \mathrm{~K}$. This technique has been designed to refine fish oil and reduce the amount of impurities and offers the possibility of obtaining a fraction in the first separator with an acidity value and a total oxidation value (TOTOX) lower than fish oil obtains without fractionation.

Extraction with supercritical fluids has become an attractive technology for obtaining fish oil rich of omega-3. The main limitation of the SFE process is the high cost on a production scale and because the raw material must be freeze-dried to reduce its humidity to values lower than $20 \%$ and keep the omega-3 unaltered.[7]

\section{Conclusions and Results}

The extraction method with supercritical fluids is very advantageous and ecological and allows the valorization of waste products as a derived raw material or as another product.

The extraction time using supercritical $\mathrm{CO}_{2}$ is shorter than the extraction time using common solvent, since $\mathrm{CO}_{2}$ has a strong permeability and, moreover, the first advantage of this technique is that, at the end of the extraction process, the carbon dioxide is brought to room temperature and atmospheric pressure and consequently gasifies, 
leaving the substances extracted from the solid matrix.

The use of $\mathrm{SC}-\mathrm{CO}_{2}$ in the extraction of lycopene from tomato skins has produced numerous advantages especially in the higher yields obtained, in the selectivity of the extraction and in obtaining lycopene without traces of organic solvents.

In fact, the extraction techniques commonly used for the extraction of lycopene from vegetable matrices use organic solvent, which, however, has two drawbacks: the first is that it is not specific for lycopene, but extracts many other pigments (carotenes, xanthophylls) with the consequence that the purification process of lycopene becomes long and expensive and in general does not lead to having lycopene of high degree of purity; the second contraindication is that the raw material used becomes a special waste that will have to be incinerated with considerable cost increase and cannot be recovered in any way.

The organic solvent that causes the least problems is ethanol, but any solvent extraction requires a subsequent stage of purification.

The extraction of fish oil by wet pressing is the most commonly used method for industrial-scale production and it is basically performed in four phases: cooking of the fish, pressing, decanting and centrifugation.

Another conventional procedure is solvent extraction: the main limitation of this technique is that it requires a relatively dry sample that is destroyed, and also takes a long time, in addition to generating large amounts of residual solvent.[9]

After fish oils are extracted, they require a purification process to achieve quality characteristics that make them acceptable for human and animal consumption, as they contain insoluble impurities.

The traditional refining process includes various phases, such as degreasing, neutralization, bleaching, deodorization and, in some cases, winterization.

Despite the need for a high investment cost, the supercritical fluid extraction of essential oils requires lower processing costs and downstream processes that make this process competitive with regards to steam distillation.

In the industrial field the application of supercritical fluids in the extraction of omega-3 should be extended to the whole process: extraction, fractionation, concentration of omega-3, in order to obtain omega-3 concentrates used in functional foods and in the pharmaceutical field.

\section{References:}

[1] Sengers, JMH Levelt, and Erdogan Kiran, eds. Supercritical Fluids: Fundamentals for Application. Kluwer Academic Publishers, 1994.

[2] Hitchen, S. M., and J. R. Dean. "Properties of supercritical fluids." Applications of Supercritical Fluids in Industrial Analysis. Springer, Dordrecht, 1993. 1-11.

[3] Reverchon, Ernesto, and Iolanda De Marco. "Supercritical fluid extraction and fractionation of natural matter." The Journal of Supercritical Fluids 38.2 (2006): 146-166.

[4] Lesoin, Laurène, et al. "Preparation of liposomes using the supercritical anti-solvent (SAS) process and comparison with a conventional method." The Journal of Supercritical Fluids 57.2 (2011): 162-174.

[5] Vasapollo, Giuseppe, et al. "Innovative supercritical $\mathrm{CO}_{2}$ extraction of lycopene from tomato in the presence of vegetable oil as cosolvent." The Journal of Supercritical Fluids 29.1-2 (2004): 87-96.

[6] Rubio-Rodríguez, Nuria, et al. "Supercritical fluid extraction of fish oil from fish byproducts: A comparison with other extraction methods." Journal of Food Engineering 109.2 (2012): 238-248.

[7] Bonilla-Méndez, Jeimmy Rocío, and José Luis Hoyos-Concha. "Methods of extraction refining and concentration of fish oil as a source of omega-3 fatty acids." Corpoica Ciencia y Tecnología Agropecuaria 19.3 (2018): 645668. 